\title{
Telemedicine and technological devices for amyotrophic lateral sclerosis in the era of COVID-19
}

\author{
Fabiola De Marchi ${ }^{1}$ (ID $\cdot$ Roberto Cantello $^{1} \cdot$ Serena Ambrosini ${ }^{2} \cdot$ Letizia Mazzini $^{1}$ • On Behalf of the CANPALS Study \\ Group
}

Received: 18 April 2020 / Accepted: 7 May 2020 / Published online: 21 May 2020

(C) Fondazione Società Italiana di Neurologia 2020

Dear Editor,

The management and monitoring of neurological patients suddenly had to change due to coronavirus disease-19 pandemic.

COVID-19, an infectious disease caused by severe acute respiratory syndrome coronavirus 2 (SARS-CoV-2), was declared a global pandemic on March 11, 2020, by the World Health Organization, after the virus spreading from China to Europe, Italy firstly.

In Italy, at the time we write this letter, 2 months after the first positive case, there are more than 197,000 infected people and more than 26,000 deaths. The authors of this communication are writing from Tertiary Regional Center for Amyotrophic Lateral Sclerosis (ALS) of Neurological Department, in Novara, Piedmont, Italy, which is the third most affected regions (roughly 24,000 cases).

In this tragic situation, the clinical neurological practices have, by necessity, changed dramatically over the pandemic diffusion. The greatest changes and difficulties derive from the need to change the approach and management of patients both with acute and, especially, chronic/neurodegenerative disorders, as well described by Guidon and Amato [1].

Fabiola De Marchi

fabiolademarchi@gmail.com; fabiola.demarchi@uniupo.it

1 Department of Neurology and ALS Centre, University of Piemonte Orientale, Maggiore della Carità Hospital, Corso Mazzini 18, 28100 Novara, Italy

2 Serena Ambrosini, Consoft Sistemi, Turin, Italy

3 Department Dietetic and Clinical Nutrition, University of Piemonte Orientale, Maggiore della Carità Hospital, Novara, Italy

4 Department of Respiratory Diseases, Maggiore della Carità Hospital, Novara, Italy

5 Department of Physical Therapy, University of Piemonte Orientale, Maggiore della Carità Hospital, Novara, Italy

6 Health Management, Maggiore della Carità Hospital, Novara, Italy
The out-patient follow-up visits for patients with chronic diseases have been suspended, maintaining only the possibility of exceptionally performing visits for urgency. In addition, the overloading of the national health system and the fear of contagion in patients who came to the hospital for nonCOVID19-related disorders have dissuaded the access to the emergency room if not strictly unavoidable.

In this context, the management of patients with a rapidly progressive disabling pathology such as amyotrophic lateral sclerosis becomes extremely complicated, because the patients can rapidly feel themselves lost due to the lack of a constant medical and psychological support. We are faced with patients who will not see their reference doctor for months, with the risk to fall into a functional deterioration due to the difficulties in therapeutic changes and the impossibility of preventing pathology-related complications. Peculiarly, ALS patients with impaired respiratory function and/or rapid clinical deterioration are a high-risk population for severe complications and also for COVID-19.

Furthermore, patients experience an understandable condition of emotional distress amplified by current external stimuli that make them more vulnerable.

In a routinely context, depending on the needs related to the individual subjects, these patients are followed and monitored with periodic outpatient visits, adding, if needed, several numbers of contacts and any other access (for patient, caregiver, or family member).

In this global situation, the need of switching to alternative type of cares, including the non-face-to-face care, is becoming mandatory to prevent a greater decline of the physical and psychological functions. It is therefore evident that the need to launch a home telecare approach in our patients becomes essential [2,3].

For patients followed at Our Center, the current practice of caring involves a multidisciplinary team consisting of neurologist, pneumologist, dietologist, physiatrist, nurse, physical therapist, dietitian, and psychologist, that the ALS patient uses to be examined every $2-5$ months, and always contactable by patients 
and caregivers. Based on this integrated and efficient approach, we theorize that satisfactory multidisciplinary care could be provided to our patients in their home using technology.

Over the last years, various telehealth technologies have been incorporated into the practice of neurology, and it is expanding to the management of a variety of chronic neurologic disorders including epilepsy, headache, movement disorders, and motoneuron diseases. Technologies that enable patients to access health services from their home include several methods, such as standard telemedicine (with video conferencing), telehealth (with remote monitoring of clinical measurements), telecoaching, and telecare (technology to support independent living), supported by health "apps" and internet-based education material [4].

The development of this essential approach will be possible thanks to the proposal "C.a.n.P. - La Casa nel Parco" for ALS, as project part of the "E-Health, Bioinformatics and ICT" for Health Research section of the "Health and Wellness Technology Platform tender" of the Piedmont Region. The C.a.n.P. project starts from a model of hospitalization called "Hospitalization at Home" that, with the use of technological devices, it tries to improve the assistance and quality of life of the monitored patients. From the technological point of view, the project deals with integrating and applying new solutions in the world of Information and Communication Technologies, which are the set of digital methods and techniques used in the transmission, reception, and processing of data.

The general aim of this project is to use technology to improve access to specialist care for patients with ALS and their caregivers, improving their quality of life. Specifically, we would like to understand what aspects of the traditional specialist ALS service could be delivered using technology, measuring the relevant impacts of this intervention in different areas. Starting from these assumptions, we are trying to apply this innovative method in patients with motor neuron diseases during COVID-19 pandemic. The primary objective during COVID-19 pandemic will be the improvement of the quality of life of the patients and the caregivers, reducing anxiety and depression related to the worldwide emergency, using a multidisciplinary approach to prevent/ monitoring the development of disease complications (e.g., malnutrition), reducing the in-person hospital care.

We set ourselves the goal of monitoring, during the months of the pandemic, the main spheres involved in ALS patients, with the cooperation of our team, who already knows the single patients: the medical, functional and the neurorehabilitative support, the psychological support, the nutritional counseling.

In consideration of the criticality and difficulty of this historical moment, regardless of the functional sphere that will be evaluated in each patient, it will be mandatory to monitor how our approach can be able to restrain clinical, functional, and psychological complications, such as anxiety and depression.

In particular, as support for patient family doctor and for home care service, additional attention will be given to the management of respiratory complications which, due to their variety, could be mixed up with Sars-CoV-2 infection.

Patients in telemedicine will also be asked to fill out an ALSFRS-R scale that we have developed to be self-administered. The main objective will be to evaluate the different perception of their own functional state of disease during and after the COVID-19 emergency, and how much this could differ from the healthcare provider's evaluation.

All the software we will use require only an online connection by personal computer of tablet. If participants will not have any device, we will provide a table for the trial period. Participants will be instructed to use the software app for telemedicine-telecoaching and telehealth. We will contact patients every 2 weeks, and we require to fill out subjective evaluation scales every month, for at least 3 months (or, until the end of the emergency situation).

The nutritional counseling will be also supported by the use of an easy online software app (which will be provided to the patient through a link) able to monitor the dietary goals, the portions of food introduced, with frequent reminders and physical/dietetic measurements, as already demonstrated by colleagues as effective method [5].

We hope to detect as soon as possible the relevance of this approach and how it can be useful to avoid, as much as possible, the postponed disease-related complications of this devastating disease, trying to support patients and their caregivers in the best way.

Acknowledgments We would like to thank Stefania Moia, project coordinator, and all study participants and their families and caregivers for their dedication and contribution to ALS research.

Funding information This project is supporting by Regione Piemonte, regional program "Investimenti a favore della crescita e dell'occupazione" - Piattaforma Salute POR/FESR 2014/2020 and by the AGING Project for Department of Excellence at the Department of Translational Medicine (DIMET), Università del Piemonte Orientale, Novara, Italy.

\section{Compliance with ethical standards}

Conflict of interest The authors declare that they have no conflict of interest.

Ethics approval The study was approval by Ethics Committee on 13/3/ 2020 (number CE19/20).

\section{References}

1. Guidon AC, Amato AA (2020) COVID-19 and neuromuscular disorders [published online ahead of print, 2020 Apr 13]. Neurology. https://doi.org/10.1212/WNL.0000000000009566

2. Klein BC, Busis NA (2020) COVID-19 is catalyzing the adoption of teleneurology. Neurology. https://doi.org/10.1212/WNL. 0000000000009494 
3. Hollander JE, Carr BG (2020) Virtually perfect? Telemedicine for Covid-19. N Engl J Med 382(18):1679-1681. https://doi.org/10. 1056/NEJMp2003539

4. Helleman J, Van Eenennaam R, Kruitwagen ET et al (2020) Telehealth as part of specialized ALS care: feasibility and user experiences with ALS home-monitoring and coaching. Amyotroph Lateral Scler Frontotemporal Degener:1-10
5. Wills AM, Garry J, Hubbard J et al (2019) Nutritional counseling with or without mobile health technology: a randomized open-label standard-of-care-controlled trial in ALS. BMC Neurol 19(1):104

Publisher's note Springer Nature remains neutral with regard to jurisdictional claims in published maps and institutional affiliations. 